Pathologe 2010 · 31:409

DOI 10.1007/s00292-010-1373-3

(c) Springer-Verlag 2010

\title{
K.W. Schmid
}

Institut für Pathologie und Neuropathologie, Universitätsklinikum Essen, Universität Duisburg-Essen

\section{Zur Übernahme der Schriftleitung}

In der letzten Ausgabe von Der Pathologe hat K.-M. Müller eine umfassende Darstellung der Entwicklung der einzigen deutschsprachigen Fachzeitschrift für das Fach Pathologie gegeben [1]. Unter seiner Leitung wurde mit tatkräftiger Unterstützung des Springer-Verlags die Gestaltung des Journals in eine allgemein geschätzte und zur Unterstützung der täglichen Diagnostik unabdingbare Zeitschrift mit fast ausschließlich edukativem Charakter vollzogen. Das Prinzip der Themenschwerpunkte wurde begeistert aufgenommen: Es bietet die einmalige Gelegenheit, einen Themenkreis umfassend unter Berücksichtigung der modernen Entwicklungen der Pathologie in prägnanter Form abzuhandeln.

Für das gesamte, inzwischen erweiterte Editorial-Board und mich ist es eine große Herausforderung, das bestehende Konzept der letzten Jahre erfolgreich weiterzuführen, aber auch das Spektrum der Zeitschrift durch die Einführung verschiedener Sparten zu erweitern und damit den hohen Standard noch zu verbessern.

Mein Dank gilt K.-M. Müller und seinen für Der Pathologe tätigen Mitarbeitern für das hervorragend bestellte Feld, das sie mir übergeben haben. Mein Dank gilt aber auch einer Reihe von Kollegen, die ausnahmslos und spontan meiner Bitte um Mitarbeit in dem von mir gewünschten erweiterten Editorial-Board gefolgt sind, sowie den bisherigen Mitgliedern der Schriftleitung, die ebenfalls gerne ihre weitere Tätigkeit in diesem Gremium unter meiner Leitung zugesagt haben.
Die Leserschaft möchte ich bitten, Wünsche, Anregungen, aber natürlich auch Kritik an die Schriftleitung heranzutragen. Der Erfolg von Der Pathologe wird auch in Zukunft an der Akzeptanz durch seine Leserschaft gemessen werden.

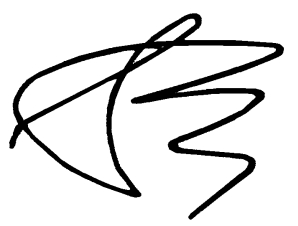

K.W. Schmid

\section{Korrespondenzadresse \\ Prof. Dr. K.W. Schmid \\ Institut für Pathologie und \\ Neuropathologie, \\ Universitätsklinikum Essen, \\ Universität Duisburg-Essen \\ Hufelandstr. 55, 45122 Essen \\ kw.schmid.editor@uk-essen.de}

\section{Literatur}

1. Müller KM (2010) Zur Übergabe der Schriftleitung. Pathologe 31:329-330 